\title{
TIC: facilitadoras de la continuidad de actividades en la contingencia de covid-19
}

\author{
Ana Aurora Vidal Martínez y Beatriz Olivia Camarena Gómez
}

\begin{abstract}
Resumen
La pandemia provocada por el covID-19 se extendió rápidamente a nivel mundial, y afectó, significativamente, a todos los países en el ámbito económico y social por los programas de confinamiento y aislamiento. En tal contexto, las instituciones económicas y de servicios se han visto en la necesidad de modificar sus formas de trabajo para facilitar la continuidad de sus actividades en y desde casa; esta situación se puede apreciar en casi todos los centros de investigación e instituciones de educación superior. El objetivo de este estudio es explorar el uso de las Tecnologías de Información y Comunicación (TIC) en un centro de investigación del noroeste de México, para facilitar la continuidad de las actividades fundamentales durante los primeros meses de este período de contingencia sanitaria. El estudio, también, hace hincapié en la necesidad de diseñar y de incorporar, de manera gradual y sistemática, nuevos tipos de modelos de trabajo que aprovechen las nuevas tecnologías de información y, además, plantea la posibilidad de rediseñar y de fortalecer, de manera más sistemática, los procesos y las gestiones administrativas, sobre todo académicas, en la modalidad a distancia.
\end{abstract}

Palabras clave: entorno virtual, educación en línea, herramientas virtuales, recursos didácticos.

\section{Challenges and PeRspectives of ICt to guarantee the ContinUity of activities IN THE COVID-19 CONTINGENCY}

\begin{abstract}
The covid-19 pandemic has spread worldwide, significantly affecting all countries in their economic and social spheres, basically due to the confinement and social isolation programs promoted. In this context, economic and service institutions have found it necessary to modify their ways of working to facilitate the continuity of their activities at and from home, a situation that can be seen in almost all research centers and higher education institutions. The objective of this study is to explore the use of Information and Communication Technologies (ICT) to facilitate the continuity of the fundamental activities of a research center in northwestern Mexico during the first months of this health contingency period. The study makes explicit the need to gradually and systematically design and incorporate new types of work models that take advantage of new information technologies and raises the possibility of redesigning and strengthening, in a more systematic way, administrative processes and management, especially academic ones. in remote mode
\end{abstract}

Keywords: virtual environment, online education, virtual tools, teaching resources.

Recepción: 04/09/2020. Aprobación: 15/03/2021. http://doi.org/10.22201/cuaieed.16076079e.2021.22.4.13 
"TIC: facilitadoras de la continuidad de actividades en la contingencia de COVID-19" Ana Aurora Vidal Martínez y Beatriz Olivia Camarena Gómez

Vol. 22, Núm. 4, julio-agosto 2021

Revista Digital Universitaria

Ana Aurora Vidal Martínez

avidal@ciad.mx

orcid.org/0000-0002-5575-3267

Google Scholar: Ana Aurora Vidal M.

Estudió la maestría en el programa "Educación Basada en Competencias", de la Universidad Valle de México. Actualmente es Investigadora Asociada B en la Coordinación de Programas Académicos, en el Centro de Investigación en Alimentación y Desarrollo (CIAD), A.C.

Le interesa la tecnología educativa, y sus áreas de investigación son la educación a distancia, el seguimiento de egresados y los sistemas de comunicación.

Beatriz Olivia Camarena Gómez

betica@ciad.mx

orcid.org/0000-0002-7417-6012

Google Scholar: Beatriz Olivia Camarena Gomez

Cuenta con estudios de doctorado en el programa "El medio ambiente natural y humano en las Ciencias Sociales" en la Facultad de Educación de la Universidad de Salamanca, España. Actualmente es sNi nivel I e Investigadora Titular C en la Coordinación de Desarrollo Regional en el Centro de Investigación en Alimentación y Desarrollo (CIAD), A.C.cxzb. ds

Está interesada en los estudios ambientales y socioculturales del desarrollo. Sus líneas de investigación son el estudio y gestión ambiental del desarrollo, economía regional y sustentabilidad ambiental, diseño y evaluación de programas y estrategias de educación y comunicación ambiental. 
"TIC: facilitadoras de la continuidad de actividades en la contingencia de COVID-19" Ana Aurora Vidal Martínez y Beatriz Olivia Camarena Gómez

\section{Introducción}

El 11 de marzo de 2020, la Organización Mundial de Salud declaró una pandemia a causa de la covid-19, al contabilizar, en esas fechas, 118000 casos de contagio distribuidos en 114 países, y un total de 4,291 personas fallecidas (World Health Organization [WHO], 2020). Para el 30 de marzo del mismo año, los números aumentaron a 756,658 de casos y más de 36,767 muertes en 194 países (Radiotelevisión Española [RTVE], 2020). En México, para atender tal situación de salud pública, se estableció la Jornada Nacional de Sana Distancia del 23 de marzo al 19 de abril de 2020 y, siete días después, tras reportar un saldo nacional de 1,094 casos positivos, 2,752 casos sospechosos y 28 fallecimientos a causa de la covid-19, se declaró una situación de emergencia sanitaria y se reforzaron las medidas de la estrategia nacional de combate, entre éstas, destaca la ampliación del período de suspensión de actividades no esenciales en los sectores públicos, privados y sociales. En consecuencia, estas medidas pusieron en evidencia la necesidad de virtualizar las actividades, sobre todo en las instituciones de educación superior y en los centros de investigación del país.

Sin duda, la presencia del SARS-CoV-2 y la situación de emergencia sanitaria pública plantean grandes retos para todos los países y exigen, particularmente, el confinamiento social y, como consecuencia, demandan una búsqueda de nuevas formas de trabajo que faciliten la continuidad de las actividades de manera remota. Para este estudio, se tomará como ejemplo el Centro de Investigación en Alimentación y Desarrollo A.c. y se explorará cómo han realizado sus actividades académicas a partir de la suspensión de las clases presenciales en todos los programas de posgrado (maestría y doctorado). Además, se observará el funcionamiento de las actividades administrativas, de investigación y de vinculación. Es importante precisar que, en el Centro de Investigación, la idea de virtualización ya estaba presente antes de la pandemia de la coviD-19, sin embargo, se veía poco factible avanzar en tal proceso en un corto periodo de tiempo; así, debido a la contingencia sanitaria, en el Centro, se pudieron enfrentar los obstáculos y se logró impulsar los cambios necesarios casi de manera inmediata para implementar la educación a distancia o la virtualidad, a pesar de los escasos recursos disponibles.

El propósito de este trabajo es explorar los cambios registrados y los resultados obtenidos hasta la fecha para lograr continuar con las actividades del Centro. Aquí, importa analizar cómo la situación de contingencia aceleró e impulsó el trabajo en y desde casa, más allá de las aulas o las oficinas. En particular, interesa describir los principales cambios suscitados en los programas de posgrado. Para realizar la evaluación del proceso de virtualización, se adoptó un enfoque metodológico cualitativo, el cual consiste en una serie de métodos y técnicas para describir y analizar las medidas que se tomaron en el Centro, con un enfoque especial en las dimensiones de accesibilidad y de disponibilidad. 


\section{Metodología}

Desde mediados de marzo de 2020 hasta la fecha, el personal de las distintas áreas del Centro ha desempeñado sus principales tareas profesionales en sus casas debido a las medidas de distanciamiento social, las cuales exigieron a la Coordinación de Programas Académicos pasar de un modelo presencial a un modelo a distancia, en consecuencia, fue necesario diseñar un plan para poder concluir el semestre en los programas de maestría y de doctorado. Este plan consistió,inicialmente, en extender el cierre de semestre, establecer lineamientos para ofrecer apoyo a los estudiantes y a los docentes, una recalendarización de clases, capacitación para docentes y estudiantes y, en habilitar cuentas y espacios virtuales para los docentes.

Entre los aspectos cruciales para lograr tales cambios, se necesitaba que la institución contará con las condiciones de infraestructura básica para que todo el personal, tanto empleados como estudiantes, pudiesen trabajar a distancia. Para lograr esto, se utilizaron los recursos informáticos disponibles que brindan apoyo en el desarrollo de actividades urgentes, como la comunicación continua entre el personal y los estudiantes, y la realización de tareas de supervisión y de evaluación. En específico, se accedió a cuatro tipos de recursos:

a) Plataformas de gestión institucional, académica, administrativa, financiera y de comunicación como Tecnoeduca, Moodle, GLPI y Portal eCIAD.

b) Redes sociales y herramientas de comunicación, por ejemplo, WhatsApp, el correo institucional y Facebook.

c) Herramientas de gestión de equipo para crear y compartir contenidos tales como, Google Apps, Asana, TeamViewer.

d) Herramientas de carácter interactivo como Blue Jeans, Meeting y Skype.

En la tabla 1, se muestra el porcentaje de utilización de estos recursos por parte de los docentes, los estudiantes y los técnicos de apoyo.

Tabla 1. Uso de recursos digitales

Fuente: elaboración propia con base en monitoreo y seguimiento de actividades realizadas a través de plataformas virtuales.

\begin{tabular}{|c|c|c|c|}
\hline \multirow[t]{2}{*}{ Recursos digitales } & \multicolumn{3}{|c|}{ Usuarios } \\
\hline & Docentes & Estudiantes & Técnicos \\
\hline \multicolumn{4}{|c|}{ Plataforma de gestión institucional } \\
\hline Tecnoeduca & $100 \%$ & $100 \%$ & $5 \%$ \\
\hline Moodle & $35 \%$ & $55 \%$ & $5 \%$ \\
\hline GLPI & $100 \%$ & $100 \%$ & $100 \%$ \\
\hline Portal ecIAD & $100 \%$ & $100 \%$ & $100 \%$ \\
\hline $\begin{array}{l}\text { Redes sociales y herramienta } \\
\text { comunicación }\end{array}$ & & & \\
\hline
\end{tabular}


"TIC: facilitadoras de la continuidad de actividades en la contingencia de COVID-19" Ana Aurora Vidal Martínez y Beatriz Olivia Camarena Gómez Vol. 22, Núm. 4, julio-agosto 2021

Revista Digital Universitaria

\begin{tabular}{|l|c|c|c|}
\hline WhatsApp & $100 \%$ & $100 \%$ & $100 \%$ \\
Correo institucional & $100 \%$ & $100 \%$ & $100 \%$ \\
Facebook & $100 \%$ & $100 \%$ & $100 \%$ \\
\hline $\begin{array}{l}\text { Herramientas gestión de equipo para } \\
\text { crear y compartir contenidos }\end{array}$ & & & \\
$\quad$ Google Apps & $100 \%$ & $100 \%$ & $100 \%$ \\
Asana & $30 \%$ & $40 \%$ & $100 \%$ \\
TeamViewer & $5 \%$ & $5 \%$ & $100 \%$ \\
Herramientas de carácter interactivo & $100 \%$ & $100 \%$ & $100 \%$ \\
$\quad$ Blue Jeans & $30 \%$ & $40 \%$ & $70 \%$ \\
Meeting & $1 \%$ & $5 \%$ & $0 \%$ \\
\hline Skype & & & \\
\hline
\end{tabular}

Aquí, interesa describir los principales cambios suscitados en las clases virtuales y en los programas académicos. El estudio se realizó a través de una investigación documental en la cual, usualmente "se consolida de manera estructurada una opción epistemológica, un propósito, una ruta metodológica y un arsenal instrumental" (Galeano, 2004, p. 55). Para la recolección de información, se utilizaron las cédulas de registro de las sesiones en Blue Jeans, debido a que, en esta plataforma, los docentes titulares de cada materia, 39 en total, realizaron la programación de sus clases, especificando los nombres de los participantes (estudiante y docentes colaboradores), correos electrónicos, fechas y horarios de cada sesión. Al término del semestre, se registró cualquier eventualidad durante el proceso de enseñanza. Asimismo, se utilizaron los siguientes instrumentos para obtener los datos necesarios: el centro de atención a usuarios y los reportes de inconvenientes e inconformidades por parte de los estudiantes.

\section{Resultados}

Al extender el tiempo del cierre del semestre académico, la primera medida interna del Centro fue continuar con el resto de las actividades académicas a distancia y determinar cómo organizarlas, además de establecer algunos lineamientos para brindar apoyo a estudiantes y a docentes con el propósito de facilitar sus respectivos procesos de comunicación y de desarrollo de actividades. En atención a la recalendarización de los planes académicos, se ajustaron y se organizaron todas las clases pendientes de cada curso al nuevo calendario para ofrecerlas en una modalidad a distancia, a través de la sala virtual Blue Jeans. Esta sala permite a los participantes conectarse fácilmente a una sesión de videoconferencia basada en la nube y con la ventaja de poder utilizar una amplia gama de dispositivos como: equipos especializados, equipos de cómputo o dispositivos móviles. 
El primer paso para empezar la transición a las clases virtuales fue habilitar cuentas para los docentes en la plataforma Blue Jeans y brindar sesiones de capacitación para su uso. Posteriormente, se realizaron pruebas desde los sitios de conexión (domicilio particular del docente) para identificar posibles problemas técnicos. Durante un breve periodo de prueba, se verificó que el envío de audio, de video y de datos fueran realizados correctamente; asimismo, se vigiló que la configuración y el funcionamiento de cada uno de los dispositivos que se utilizarían para realizar las clases en línea fuera exitosa.

Para capacitar al docente en la modalidad a distancia, al principio de cada clase se le acompañó para orientarlo y para apoyarlo en la utilización de dicho sistema y en caso de que se presentará algún problema. Poco a poco, en el transcurso de las clases, el docente involucrado en tal experiencia avanzó en su proceso de capacitación y logró el aprendizaje esperado, es decir, fue capaz de continuar la comunicación a distancia sin apoyo técnico, y pudo crear sitios de gestión de recursos educativos; esto fue de mucha importancia, ya que la función principal del maestro es que el alumno atribuya significado al contenido que debe aprender.

Cada uno de los sitios creados en la plataforma educativa debía contener, obligatoriamente, los 3 tipos de módulos que se muestran en la figura 1.

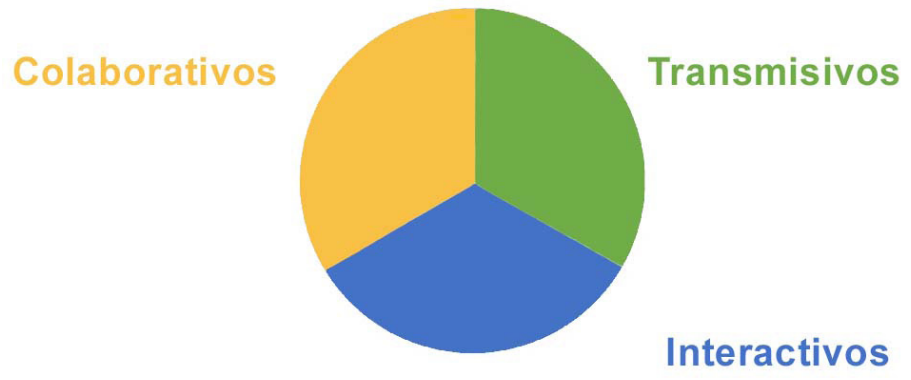

Con los estudiantes, las pruebas de conexión se supervisaron a distancia y sólo en algunos casos se brindó una orientación personal debido al tiempo limitado de cada clase y a la gran cantidad de estudiantes. Para facilitar el proceso de capacitación, se optó por elaborar breves guías de instrucciones y se facilitaron los datos del personal técnico especializado (soporte técnico) para que se les contactará en caso de ser necesario.

Cuando las coordinaciones entraron en cuarentena, se incorporaron dos herramientas más para la colaboración a distancia: Google Apps y Asana. Ambas forman parte del modelo software como servicio, los cuales ofrecen aplicaciones basadas en la nube a los usuarios por medio de un navegador web o de una interfaz de programa. Google Apps permite crear y editar documentos en tiempo real, además, admite la creación de carpetas compartidas, y también, sirve para organizar y compartir calendarios. Asana, por su parte, es otra herramienta 
"TIC: facilitadoras de la continuidad de actividades en la contingencia de COVID-19" Ana Aurora Vidal Martínez y Beatriz Olivia Camarena Gómez Vol. 22, Núm. 4, julio-agosto 2021 Revista Digital Universitaria

gratuita, esta permite realizar un seguimiento de trabajos colaborativos debido a que facilita la realización de colaboraciones en tiempo real y permite compartir informes y actualizaciones del estado de actividades y de proyectos. Al igual que en el área de posgrados, las diferentes coordinaciones hicieron uso de la sala virtual Blue Jeans para llevar a cabo las reuniones de trabajo.

Como otros ejemplos de creación de programas y estrategias de aprendizaje en línea, está Margolis, quien realizó un webinar donde la mayoría de las actividades se diseñaron para ser asíncronas, es decir, los inscritos no requerían ni una fecha ni una hora específica para poder participar. Dentro de estas actividades, se incluyeron conferencias magistrales, simulaciones clínicas y foros de discusión. La interacción entre participantes y docentes se llevó a cabo por una plataforma similar a Facebook en donde se aprovechan las relaciones personales para generar confianza y fomentar un diálogo significativo (2020).

En la misma línea, Castillo concluye que debemos de aprovechar las oportunidades que nos brinda la pandemia de la coviD-19, ya que permitió notar la necesidad de contar con un sistema educativo con capacidad para adaptarse a los cambios y con conocimientos sobre el uso de los medios digitales y tradicionales, como la televisión o la radio. Finalmente, reflexiona que las capacidades de los y las docentes se fortalecieron y que deben ser consideradas como parte de las nuevas metodologías de enseñanza en las aulas (2020). Al respecto, Fernández y Mella consideran que el avance de las Tıc, en estos tiempos pandémicos, da lugar a un panorama en la que todos nos convertimos en coeducadores y en coaprendices debido a la construcción colectiva de conocimiento que está sucediendo (2016).

En lo que compete a la experiencia educativa virtual, es ilustrativo el aumento del número de clases a distancia brindadas tan sólo en la coordinación central al iniciar la crisis sanitaria. En la tabla 2 se puede observar dicho aumento, ya que durante el primer período comprendido del 20 de enero al 16 de marzo del presente año (inicio y desarrollo del semestre hasta antes de la contingencia sanitaria) se registraron menores sesiones y planificaciones de salas virtuales que en el periodo del 17 de marzo al 26 de mayo del presente (inicio de clases en modalidad a distancia hasta conclusión del semestre).

Tabla 2. Comparativo de actividades educativas a través de plataformas virtuales antes y durante etapa de confinamiento por SARS-COV-2.

Fuente: elaboración propia con base en monitoreo y seguimiento de actividades realizadas a través de plataformas virtuales.

\begin{tabular}{|l|l|l|l|}
\hline \multicolumn{1}{|c|}{ Actividades / Procesos } & \multicolumn{1}{c|}{$\begin{array}{c}\text { Antes de } \\
\text { Confinamiento }\end{array}$} & $\begin{array}{c}\text { Perído } \\
\text { Confinamiento }\end{array}$ & \multicolumn{1}{c|}{ Fuente } \\
\hline Clases a Distancia (CDH) & 14 & 39 & $\begin{array}{l}\text { Indepnet Association } \\
\text { Use GLPI }\end{array}$ \\
\hline $\begin{array}{l}\text { Planificadores en la sala } \\
\text { virtual }\end{array}$ & 17 & 30 & $\begin{array}{l}\text { Blue Jeans } \\
\text { Administrador } \\
\text { Usuarios. }\end{array}$ \\
\hline $\begin{array}{l}\text { Comparativo sesiones } \\
\text { realizadas en la sala virtual }\end{array}$ & $\begin{array}{l}199 \\
\text { (enero 20 a 16 } \\
\text { marzo 2020) }\end{array}$ & $\begin{array}{l}429 \\
\text { (marzo 17 a 26 } \\
\text { mayo 2020) }\end{array}$ & $\begin{array}{l}\text { Indepnet Association } \\
\text { Use GLPI }\end{array}$ \\
\hline
\end{tabular}


Es importante resaltar que, tanto empleados como estudiantes, se enfrentaron a algunos problemas de conectividad y compatibilidad con sus equipos y sus softwares, lo cual, ocasionó algunos retrasos y, en ocasiones, se vieron imposibilitados para realizar ciertas actividades. Estos problemas fueron resolviéndose gracias a los técnicos de apoyo y, para lograr superar esta situación, se realizaron capacitaciones personalizadas, asistencia técnica por medio de aplicaciones de control remoto para los equipos y, en algunos casos, fue necesario acceder a los equipos presencialmente, siempre tomando las medidas de protección establecidas en los protocolos.

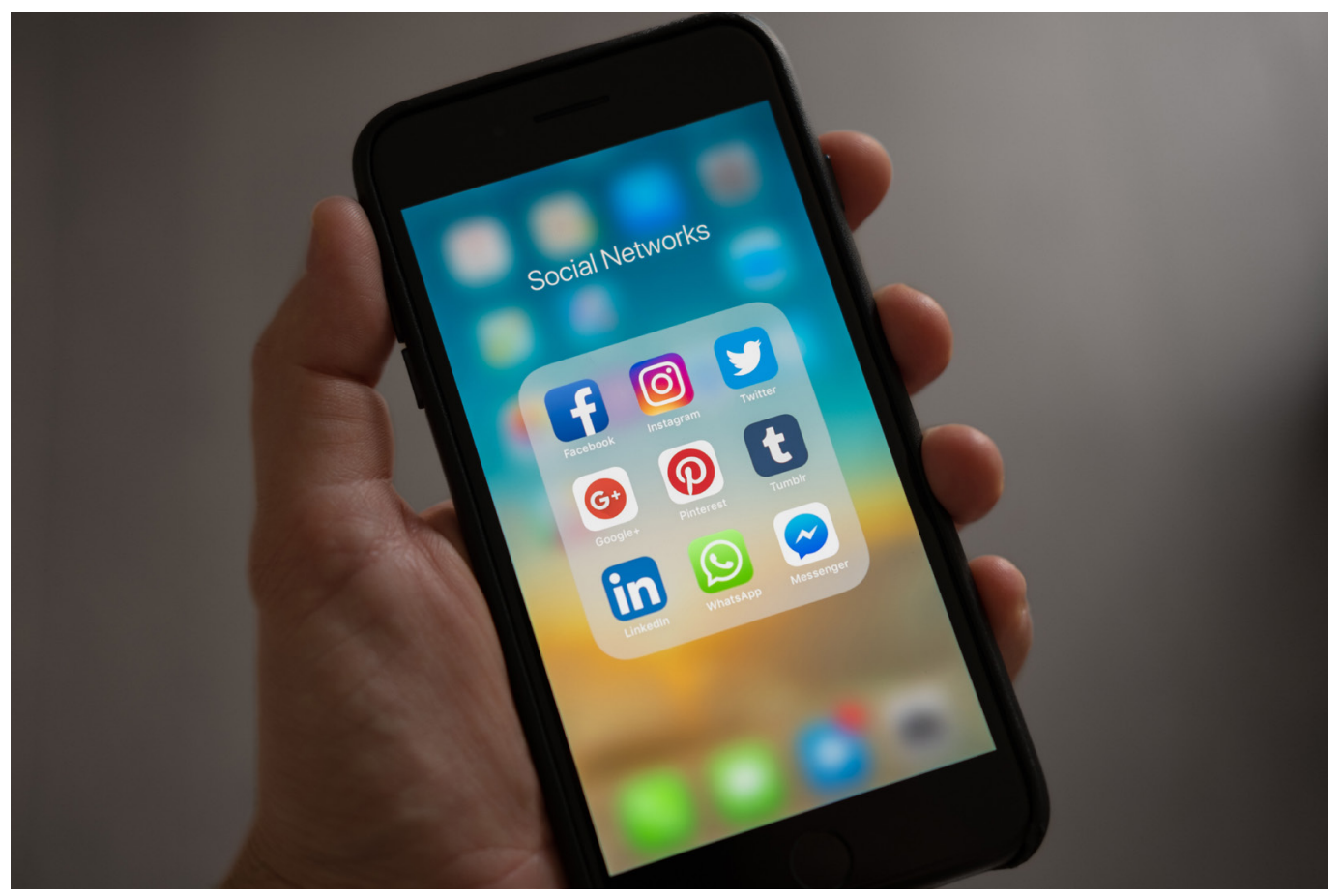

\section{Conclusiones}

Para finalizar, primero, se reconoce la rapidez con la que los docentes se adaptaron a la modalidad a distancia, y con la cual se convirtieron en usuarios de un gran abanico de recursos y de diversas formas de enseñanza. Al respecto, Morata concluye en su investigación que "las orientadoras y los orientadores no ignoran estos cambios y por lo mismo tratan de integrar en su día a día las tecnologías en su función profesional" (2016). Más allá de la crisis del coronavirus, nos encontramos inmersos en una sociedad que vive en un permanente estado de revolución y de cambio tecnológico. Rozo García, por su parte, establece que la era de la digitalización afecta nuestras formas y nuestros modos de vida, la manera en que trabajamos y en la que nos relacionamos (2020), en estos momentos de contingencia, la digitalización embona como anillo al dedo. 
"TIC: facilitadoras de la continuidad de actividades en la contingencia de COVID-19"

Ana Aurora Vidal Martínez y Beatriz Olivia Camarena Gómez

Vol. 22, Núm. 4, julio-agosto 2021

Revista Digital Universitaria

Un recurso importante que facilitó este proceso de ajustes y de cambios para hacer viable el trabajo a distancia, fue la sala virtual Blue Jeans. Este recurso permitió continuar con la parte teórica de las clases y, con ello, todos los estudiantes lograron proseguir con su educación. Sin embargo, referente a las prácticas de laboratorio, estas no han podido realizarse, por lo que vemos necesario trabajar en los planes de estudio y en probar nuevos instrumentos y modelos.

Por otra parte, aun cuando en algunas asignaturas se utilizó la plataforma Moodle para la creación de los aprendizajes en línea (Sánchez, 2009) y para la gestión de los contenidos de los cursos, consideramos que tal plataforma podría emplearse en todas las demás asignatura sin embargo, esto no fue posible por la falta de tiempo y preparación, pero resulta muy interesante la propuesta de esta plataforma.

Referente a las actividades administrativas, de investigación y de vinculación, estas se han realizado a la distancia mediante la utilización de diversos recursos informáticos. Las experiencias han mostrado que es posible trabajar a distancia y que se puede obtener resultados positivos. Sin embargo, una vez concluido el periodo de contingencia sanitaria, las preguntas que valdrá la pena plantear serán: ¿cuál será el futuro de la educación a distancia?, ¿se brindará continuidad y seguimiento a los procesos de virtualización iniciados como un medio de comunicación complementario para reforzar las actividades profesionales desempeñadas de manera habitual? O bien, ¿se regresará a las prácticas anteriores abandonando los esfuerzos de educación a distancia y de la comunicación virtual iniciados?

\section{Referencias}

* Asana. (2020). Software de administración y seguimiento para equipos de trabajo [aplicación web]. Google Play Store. https://play.google.com/store/apps/ details?id=com.asana.app

* Blue Jeans. (2017). Getting started guide [presentación de diapositivas]. Blue Jeans. https://www.bluejeans.com/sites/default/files/Blue-Jeans-Getting-Started-Guide. pdf

* Castillo Aramburu, M. (2020). Opciones para la actividad escolar durante la pandemia, covid-19 el caso de Nicaragua. En J. A. Huete Pérez (Ed.), Aportes para enfrenar la pandemia (21-26). Academia de Ciencias de Nicaragua.

* Fernández, C. y Mella, I. (2016). Percepción y uso de las tıc en la formación continua en las spin-off de la Universidad de Santiago de Compostela (usc). Píxel-Bit. Revista de Medios y Educación, (49), 9-23. https://recyt.fecyt.es/index.php/pixel/article/ view/61711

* Galeano, M. E. (2004). Diseño de proyectos en la investigación cualitativa. Fondo Editorial Universidad EAFIT. 
"TIC: facilitadoras de la continuidad de actividades en la contingencia de COVID-19" Ana Aurora Vidal Martínez y Beatriz Olivia Camarena Gómez Vol. 22, Núm. 4, julio-agosto 2021 Revista Digital Universitaria

* GLPI. (2013, 9 de agosto). gLPI 0.84 AVAILABLE. https://glpi-project.org/glpi-0-84available/

- Margolis, A. (2020). ¿Cómo migrar de un congreso tradicional a un congreso semipresencial o totalmente virtual? Un caso de estudio de éxito frente a la pandemia Covid-19. Revista Colombiana Nefrolofía, 7(Supl 2). https://doi.org/10.22265/ acnef.7.supl.2.415

- Morata Sanz, J. I. (2020). Uso de tic en orientación educativa en tiempos de covid-19. Revista AOSMA, 88-91. https://dialnet.unirioja.es/servlet/articulo?codigo=7381638

* Rozo García, F. (2020). Revisión de las tecnologías presentes en la industria 4.0 Revista uIs Ingenierías 19(2), 177-192. https://doi.org/10.18273/revuin.v19n22020019

* Radiotelevisión Española (RTVE). (2020, 30 de marzo). Mapa del coronavirus en el mundo: casos, muertes y los últimos datos de su evolución. https://cutt.ly/MnHmfkO

* Sánchez Rojo, I. J. (2009). Plataforma Educativa Moodle: Administración y Gestión. Alfaomega/Ra-Ma.

* World Health Organization (wHo). (2020, 11 de marzo). wHo Director-General's opening remarks at the media briefing on covID-19. https://cutt.ly/XnHmkaL

\section{Cómo CITAR ESTE ARTículo}

* Vidal Martínez, Ana Aurora y Camarena Gómez, Beatriz Olivia. (2021, julioagosto). Las tic para garantizar la continuidad de actividades ante la contingencia de covid-19. Revista Digital Universitaria (RDU), 22(4). http://doi.org/10.22201/ cuaieed.16076079e.2021.22.4.13 